\title{
Telepsychiatry: An Electronic Alternative
}

\author{
Shinu Kuriakose* \\ Department of Physician Assistant Studies, USA
}

*Corresponding author: Shinu Kuriakose, Department of Physician Assistant Studies, USA.
Received Date: September 27, 2019

Published Date: October 30, 2019

\begin{abstract}
One of the major issues facing the patient population in the United States is the problem of effective and timely mental health care. The core of this issue is that patients are unable to see a mental health provider in a timely manner to be screened for psychiatric illness, be competently diagnosed and subsequently treated in an evidence-based manner. In a bid to mitigate this issue, the field of telepsychiatry has slowly evolved in the last couple of decades into a system which allows face-to-face quality psychiatric assessment, diagnosis and treatment modalities delivered through electronic mediums, primarily video-teleconferencing. This electronic, cost effective mechanism allows access to a mental health provider in a timely manner, enhancing psychiatric care due to early intervention and follow-up, leading to positive outcomes. There is a dire need for mental health providers to be taught the skills required to evaluate patients who are not geographically present in their vicinity due to various circumstances and the optimal ways to assess their mental health conditions. There is a need for clinicians to learn this new and evolving technology and gain expertise to identify underlying emotional issues via technological means and help patients in achieving positive mental health outcomes.
\end{abstract}

Keywords: Psychiatry; Telepsychiatry; Electronic diagnosis; Mental health challenges; Positive psychiatric outcomes; Telepsychiatry challenges

\section{Introduction}

One of the major issues facing the patient population in the United States (US) is the problem of effective and timely mental health care. As we have, unfortunately, seen in the last few decades, there have been a myriad of socially devastating events occurring in our schools, workplaces, campuses, and other public areas where individuals are harming their fellow humans, and it all boils down to the perpetrators being mentally ill and thus hurting others. It is estimated that $26.2 \%$ of Americans over the age of 18 meet the criteria for a psychiatric disorder with $6 \%$ of this population being classified as severely mentally ill, a condition affecting their quality of life [1]. The core of this issue is that these folks were unable to see a mental health provider in a timely manner to be screened for psychiatric illness, be competently diagnosed, and subsequently treated in an evidence-based manner [2]. Approximately 56\% of adults in the United States with mental health maladies have been unable to receive treatment in a timely manner with certain states only having one psychiatric provider per 1,000 individuals [1]. Additionally, $8.47 \%$ of the US population suffers from alcohol and/ or drug dependence without access to timely and effective care [1]. Often, we hear stories from their family members that these patients have been progressively decompensating for a relatively long period (weeks to even years) and were unable to be seen by a psychiatric provider due to lack of clinicians in the area; financial cost of seeing providers, which the patients could not overcome; and the fact that this discrepancy has also led to a supply and demand problem: providers only seeing patients who pay cash and not accepting private and public insurance because the cash pay for service is so much more lucrative [2].

This psychiatric problem in the US has engulfed this country into a crisis in the mental health field, an issue, which not only deals with internal family problems in having patients see providers in a cost-effective timely manner, but also external disparities about having mental health providers be accessible within a reasonable geographical distance. This mental health crisis has also been exacerbated by the decrease in the number of psychiatrists from 37,968 to 37,889 which is a $10.2 \%$ reduction in the amount of psychiatrists per 100,000 people from 2003-2013 [2]. Additionally, the stigma associated with societal judgment regarding a patient receiving psychiatric care has influenced patients, including war veterans suffering from Post-Traumatic Stress Disorder (PTSD) in seeking care [3]. Moreover, families are also reluctant to encourage their loved ones from accessing much needed mental health 
treatment due to concern about its long-term social impact on a patient and their career implications [3]. It is a grave situation due to the fact that this deadly combination has caused lethal repercussions for not only the individuals involved but also society at large leading to disturbing consequences.

In a bid to mitigate this issue, the field of telepsychiatry has slowly evolved in the last couple of decades into a system, which allows face-to-face quality psychiatric assessment, diagnosis, and treatment modalities, delivered through electronic media primarily video-teleconferencing [4]. This electronic, cost effective mechanism allows access to a mental health provider in a timely manner, enhancing psychiatric care due to early intervention and follow-up, leading to positive outcomes. There has been an increased buy-in of this modality by psychiatric clinicians, nurses, social workers, practice administrators, mental health organizations, and hospital executives [4].

\section{Literature Review}

\section{Telepsychiatry history and current application}

The advent of telepsychiatry occurred in the early 1960s at Dartmouth University, which pioneered a study in which mentally ill patients were evaluated by psychiatric providers via small black and white television screens with poor audio quality [5]. The ostensible purpose of this experiment was to allow patients to seek and receive care in their own community versus being transferred to a psychiatric facility 27 miles away to be evaluated. This process was further advanced by allowing psychiatrists to not only allow evaluation, but to proctor therapy groups and provide supervision and collaboration with primary care community physicians [5]. The term, telepsychiatry (meaning interaction between mental health provider and patient via a two-way television system), was coined in 1973 to better illustrate this process and to make it easily understandable to both laymen and the medical workforce. Although this technology was prohibitively expensive in its infancy due to limited availability of electronic resources, advances in this technology in the 1980s to the early part of the 21st century made telepsychiatry more cost effective and it was increasingly seen as a viable method to allow not only care for acute treatment of psychiatric emergencies, but on an ongoing basis provide therapeutic evaluation and treatment of patients in geographically distant areas and/or with physical disabilities, which made travel a grave hardship [6].

In 1993, the American Telemedicine Association was formed with a goal to "promote professional, ethical and equitable improvement in health care delivery through telecommunications and information technology" (ATA.org). The early adopters of telepsychiatry included states with sparse population located in areas, which did not have the infrastructure or resources to allow for a standalone psychiatric specialist but was able to corral this new electronic medical medium to its advantage leading to positive mental health outcomes in its population. The unique field of psychiatry with its reliance of subjective patient history mostly via patient/provider interaction helped ease the transition of other fields of medicine into this electronic sphere. A combination of clinical anecdotal reports at conferences and research articles coupled with the relatively low cost of Internet technology led this telepsychiatry initiative to flourish, and providers and other stakeholders started viewing telepsychiatry as a serious medium, which could lead not only to timely mental health access but was also cost effective [6].

In a study on prisoners in a correctional facility, which is an area of healthcare historically understaffed, mental health care delivered to correctional psychiatric patients reported no difference in receiving care via electronic mediums compared to conventionally being evaluated by a prison psychiatrist [7]. Prisoners in this study were broken down into four groups which approximated the same number of patients and their psychiatric care was broken down to telepsychiatry, telepsychology, face-to-face psychiatry and face-toface psychology and subsequent results of this study showed not only equal satisfaction among the prisoners in all four modalities of care but equitable mental health outcomes [7]. In California, the utilization of telepsychiatry in the state prison system has saved $\$ 400$ million dollars in the last decade for the state [8]. Currently, the field of telepsychiatry has evolved into a well-respected legitimate modem of psychiatric care where patients are screened, evaluated, treated, and followed up on a regular and timely basis within a framework of economic austerity and without the stigma of secondhand medical care. Additionally, the success seen in preliminary innovative approaches in this filed has led to training programs which aim to redistribute psychiatric providers in a manner where the need is the greatest. Furthermore, there are novel approaches in using algorithm-based scenarios to train primary care providers to provide initial screening and treatment of patients with mental health issues [9].

Increasingly, there is increased impetus to deliver mental health care in the privacy of patient's own home and in communities with limited access to psychiatric care or geographical barriers. This modality of care has evolved from big institutional provider (Veterans Affairs Hospitals), which were termed "clinically supervised settings" to private psychiatric providers providing mental health treatment from their own individual offices or homes, which were called "clinically unsupervised settings" [10]. A study in 2006 showed that over the course of an 11-month period, there was a decrease of $\$ 25,000$ when the modality of telepsychiatry was used compared to conventional face-to-face treatment [10]. The force which is driving this type of electronic mental health treatment has been credit to patients and clinician's familiarity with electronic devices, as witnessed by the boom of social media outlets [10].

The emphasis on delivery of competent care in a timely manner taking into account cost saving measures has also been vital in the development of telepsychiatry, a process which reduces expenses associated with travel, early treatment preventing longer hospital stays, and mitigating the costs associated with coordination of care 
Myers K \& Pruitt D [4] There are unique aspects of mental health care delivered from geographical distances, which must take into account medical malpractice liability issues, written consent of patients to be treated via this novel mechanism, orientation of both clinicians and patients to the intricacies of using a computer screen with audio capabilities, and the need for psychiatrists (in non-veterans affairs hospitals) requiring to be licensed in the state their patients are treated [10]. Furthermore, there are still questions about the validity of recording telepsychiatry encounters and if those episodes are part of a patient's medical records and subsequently the ownership of the medical records need to be clarified [10]. As the field of psychiatry often depends on the body language of patients, increasingly there has been advancement and guidelines being established on the resolution of computer screens, which are utilized to evaluate patients and transmission speeds of at least 384 kilobytes per second, is deemed as the minimum speed to prevent computer lagging [10].

\section{Telepsychiatry Definitions}

\section{New York state telepsychiatry definition}

The Office of Mental Health of the State of New York (my home state) defines telepsychiatry as "the use of two-way real timeinteractive audio and video equipment to provide and support clinical psychiatric care at a distance. Telepsychiatry services can be utilized for assessment and treatment services provided by physicians or psychiatric nurse practitioners (NPP) from a site distant from the location of a recipient" [11].

\section{American telemedicine association telepsychiatry definition}

The American Telemedicine Association (ATA) formally defines telepsychiatry as "an intentionally broad term referring to the provision of mental health and substance abuse services from a distance. Mental health is particularly suited to the use of advanced communication technologies and the Internet for delivery of care. By using advanced communication technologies, mental health professionals are able to widen their reach to patients in a costeffective manner, ameliorating the mal distribution of specialty care" [12].

\section{The American psychiatric association telepsychiatric definition}

The American Psychiatric Association (APA) states that telepsychiatry "broadly refers to innovations in information and communication technology. It is a broader concept that telemedicine/ telepsychiatry which involves providing typical care via connecting a provider with a patient across geographical distances" [13]. The multiple definitions of telepsychiatry encompasses various electronic avenues (video, audio, teleconference via phone, tablets, computers, Internet based, and electronic app based) by which mental health clinicians can provide care for psychiatric patients in a manner, which is for all intents and purposes as effective clinically as conventional care in a one-to-one setting.

\section{Need/indication}

Traditional psychiatry had its pitfalls, including limited availability of clinicians who can evaluate only certain number of patients due to staffing and distance barriers, poor adherence rates due to inadequate follow-up, feelings of shame on the part of patients due to seeing a familiar clinician in their own community, and inconsistency in being evaluated by multiple mental health providers during follow-ups [5]. Furthermore, there was limited psychiatric care during non-peak times, and wastage of time is apparent due to excessive time spent journeying to sites and spending time in waiting rooms. Finally, there was also the problem of patients with physical disabilities not being able to see a psychiatric provider in a timely fashion [14]. It has been estimated that up to $30 \%$ of the world population will suffer from mental illness at some point in their lives and two thirds of this group will be unable to access psychiatric help [14]. In the US, 57.7 million patients will need psychiatric care on an annual basis and the vast majority would not be able to fulfill this need in a timely and appropriate manner while almost 1 in 5 children in the US suffer from a mental disorder without adequate treatment [14]. Moreover, $3.5 \%$ of men and $4 \%$ of women are unable to be gainfully employed in the United States due to mental health issues they face [14]. According to the World Health Organization (2010), about 75$85 \%$ of patients in the developing world will be unable to receive psychiatric care, which is part of the $80 \%$ of patients with mental health illness living in the developing world.

\section{Advantages}

One of the biggest advantages in using this electronic medium was the fact that patients could be seen relatively soon by a clinician without considering geographical barriers and time constraints [15]. Furthermore, it mitigated costs of having a physical space to see a patient, having less embarrassment on the patient's part due to not knowing the clinician in the community, allowing patients to be evaluated by clinicians speaking their same language without having an interpreter present, having the ability to cover the usual psychiatric providers when they were on vacation/sick leave/disability, and screening patients for alcohol/drug abuse without concern about patient travel methods due to disability [15]. A comprehensive study in a California institution utilizing the telepsychiatry medium demonstrated that "higher proportion of the telepsychiatry appointments was kept (92\% telepsychiatry vs. $87 \%$ non-telepsychiatry); telepsychiatry appointments were significantly less likely to be cancelled by patients (3.5\% vs. $4.8 \%$ ) and significantly less likely to be no-shows (4.2\% vs. 7.8\%)" [16]. The state of North Carolina, funded by a pilot study by the Duke Foundation, released the results of a pilot program focused on rural areas of North Carolina where patients with psychiatric problems were evaluated by psychiatrists via telepsychiatry means. This study demonstrated that patients who were evaluated via this medium experienced shorter hospital stays and yielded savings of $\$ 1,500$ per patient, and these patients were also more than $200 \%$ likely to follow up for aftercare (US News, 2015). 
The reasons for this dramatic increase in follow up included

- $\quad$ ease of patients by being evaluated in their homes for follow up.

- timely evaluation in the emergency room where most psychiatric patients initially visit when faced with mental health symptoms.

- greater responsiveness to clinician recommendations due to the fact that mental health patients have the perception of not feeling victimized when no real-life person is present in the same room physically while their symptoms are reported (US News, 2015).

Additionally, the fact that conversations could be recorded also provided ammunition to clinicians to help admit patients who might be gravely disabled without the patient not altering his or her story afterwards and the fact that this modality could also be effectively used for group therapy among patients with similar symptoms [6]. Finally, the cost effective mechanism of telepsychiatry was a boon to financially strained community health centers due to the fact that patients could be screened at a distance for admissions and treatment and atmospheric weather or other "acts of God" not playing a tangible part in preventing treatment [5]. When used by seasoned professionals, the telepsychiatry modality has also demonstrated success in instances where children need to be evaluated by a mental health clinician, and there has not been any difference in health care outcomes noted between traditional psychiatric adolescent screening and electronic screening [9].

Beyond the traditional benefits which help in enhancing mental health outcomes in patients served by tele-psychiatric modalities,

there has been increased benefits seen in

- enhancing mental health care without regard to geographical boundaries and socioeconomic status;

- $\quad$ enhancing compliance due to reduced travel costs and less time spent on this endeavor.

- $\quad$ increasing the education and comfort level of clinicians using this electronic means of diagnosis and treatment.

- greater coordination among health care professionals and systems due to data and information being communicated in an easier and more efficient manner.

- $\quad$ increasing the likelihood of mental health clinicians serving patients in rural and underserved areas, which would also qualify them for student loan mitigation programs.

- mitigating the stigma associated with seeking and receiving mental health care [1].

\section{Challenges}

In spite of the positive aspects this technology offers, there are challenges, which need to be overcome, such as difficulty in assessing body language in patients, which is essential in diagnosing individuals for mental health purposes, and this technology does prohibit personal contact with patients (hand on shoulder, etc), which enhances greater trust between provider and patient. There have been concerns that utilizing telepsychiatry in institutions where there is not enough clinician training and feedback does lead to lower morale due to less patient interaction [16]. Furthermore, telepsychiatry remains dependent on a robust electronic communication system for thorough vocal and visual cues, which can be expensive to maintain, and the orientation to this technology does require time while also requiring financial resources to be spent for the initial investment costs to start this program [17].

There continues to be a stigma also associated with clinicians who decide to specialize in psychiatry, especially in developing countries as evidenced by $82 \%$ of Saudi Arabian medical students and $54 \%$ of Romanian medical students shunning the field of psychiatry as they were concerned that they themselves would be labeled as having a mental health issue [18]. Among medical students in Nigeria, $20-40 \%$ also felt that there were supernatural underpinnings in persons with mental health illness and they had somehow brought this disease upon themselves [18]. Additionally, a certain subset of patients suffering from paranoid tendencies might feel this emerging technology as quite intrusive and feeding upon their paranoia of being watched all the time. Moreover, there have been concerns raised about the confidentiality of telepsychiatry and the fact that this information can be stored and be utilized for nefarious purposes by other organizations, and the record of such interaction can follow the patient all his or her life [8]. Finally, there continues to be concerns about Health Insurance Portability and Accountability Act (HIPAA) violations due to fears of hacking via the electronic medium.

\section{Economics}

The fundamental reasons for the shift from traditional office and hospital-based psychiatry to increasingly utilize electronic means for mental health evaluation, screening, and treatment not only has to do with access to psychiatric care but also has serious financial underpinnings [5].

It is certainly cost effective to have a clinician see patients without the costs involved in

- $\quad$ commuting to various physical locations where patients are located, and it can be especially cumbersome when it involves journeying to rural or geographically isolated areas.

- maintaining an office structure, which frequently involves furniture, electric, and other infrastructure bills and maintaining an office staff.

- maintaining a roster of psychiatrists and other mental health provider on a specific institutions payroll if they do not have enough patient population to be evaluated and thus be a money loser proposition to this entity.

- $\quad$ reducing the cost for patients due to not having to spend money on travel and able to minimize work disruption costs by not spending that time travelling. 
- $\quad$ easier access for patients with disabilities to be evaluated by mental health clinicians without associated costs of using specialized vehicles for travel reasons.

- decreased usage of staffing hospital emergency rooms with psychiatrists on premises or on call due to the fact that contracting with telepsychiatry agencies are available 356 days a year, 24 hours a day, which would result in significant savings for a hospital's bottom line and free up medical physicians to treat more medically ill patients [19].

\section{Methodology and Procedures}

\section{Methodology}

There was only one method to be employed for this independent study. The method was developmental.

\section{Procedures}

Search procedures: A review of the significant literature related to telepsychiatry and mental health outcomes was conducted. The review highlighted the following topics: historical data, psychiatry challenges, telepsychiatry opportunities, mental health outcomes, accessibility of psychiatric care.

Libraries used: There was only one library used for the search for sources for this project. The Health Sciences Library at Stony Brook University was used for this project.

Search engines and databases used: The following databases were used to search for the initial sources for this project. The databases were Pub Med, Google Scholar and Trip Database.

Search terms: Several search terms were used to identify sources for this project. The search terms included psychiatric care, telepsychiatry, timeliness of psychiatric treatment, mental health care in disabled patients, and electronic methods of diagnosing.

Boolean strings: Boolean strings were considered for the literature search. Several Boolean strings were used: mental health AND treatment, psychiatry AND electronic media, telepsychiatry AND screening, and telepsychiatry AND mental health outcomes.

Age of the sources: The significant literature was reviewed. Sources from the last 10 years were considered for inclusion in the review of the literature. Pertinent historical or seminal articles were also considered

Inclusion criteria: There were four inclusion criteria. Inclusion criteria included

- literature published since 2005, except historical sources.

- $\quad$ peer-reviewed articles.

- Web sites relating to telepsychiatry.

- full-text articles.

Exclusion criteria: There were four exclusion criteria. The exclusion criteria included

- literature published before 2005, except historical sources.
- $\quad$ articles not peer reviewed.

- Web sites not relating to psychiatric care and treatment.

- $\quad$ articles not full text.

Research modalities: Multiple research modalities, including descriptive, observational, longitudinal, qualitative, and randomized clinical trials, are available in the literature. Research studies regarding mental health care are common and were examined. Descriptive articles emphasizing the importance of telepsychiatric modalities, mental health patient accessibility, and economics of telepsychiatry care was featured. Exploratory articles were included in the review. Various approaches to care of this special population were discussed.

\section{Discussion}

Over the last several decades, there has been an increased initiative to advance medical care in a manner where it can be cost effective, be relatively easy to access, be grounded in a foundation of evidence-based medicine and provide satisfactory health outcomes to the population at large. There have been several attempts to mitigate poor health outcomes by increasing the number of medical schools, initiate programs to teach other providers the basis of medicine to practice (physician assistants, nurse practitioners, etc.), provide subsidies and grants to institutions, which would focus their initiatives to target patients who suffer from health care disparities, which not only will include race, gender, socio-economic discrepancies, and providing loan forgiveness to practitioners practicing in areas where care is sorely needed [17]. The concept of using an electronic medium to reach out to patients who not only suffer from geographical barriers to care but also from physical disabilities, which prevent mobility or other cognitive challenges has proven to be successful in the last 50 years [19]. The advantages can be measured in objective manners, such as cost saving to both communities and institutions, decreased reliance on economically challenged communities to fund a specialized practitioner without a base to build a financially sound business, increased patient satisfaction due to ability to seek care faster, enhanced provider satisfaction partly due to being able to evaluate patients in the comfort of the providers own office, and spending less money on infrastructure to manage patient population via examining rooms [19]. Of course, challenges, such as less physical interaction between patient and provider, initial start-up investment in the electronic tools for telepsychiatry, reluctance on part of some clinicians, and patients to learn how this technology works, do loom [8].

The American Psychiatric Association (APA) has worked on developing a Telepsychiatry Toolkit which not only encompasses the physical and logistical aspects of providing mental health care but also attempts to enlist evidence-based research in its initiatives [20]. The APA advocates an approach, which not only is based on the fundamentals of patient confidentiality while providing mental health treatment but also emphasizes the preferences of the patient and the judgment of the psychiatric clinician. This approach is rooted in the fundamental belief the healthcare outcomes in telepsychiatry should be equivalent to conventional psychiatric care 
[21]. The APA also encourages reimbursement for this electronic modality at the same rate as face-to-face treatment in an attempt to encourage practitioners to embrace this mode of treatment and increase psychiatric access [21].

The American Telemedicine Association (ATA) has implemented practice guidelines while providing medical care via electronic modalities.

These guidelines include

- $\quad$ ensuring the identity and verification of both the provider and patient.

- maintaining adequate documentation of care provided while also including contact information for both parties with clear expectation discussed prior to treatment.

- the indication of telemedicine treatment if adequate clinical providers do not present physically to provide medical care.

- $\quad$ adhering the established concepts of informed consent, safety of the environment where care is being delivered and provided and follow up communication with the patient's providers as deemed necessary.

- monitoring situations which may require emergent care and having resources and referrals to provide patients with such information if needed.

- $\quad$ ensuring that all ethical and professional standards are met while proving care via telehealth means;

- $\quad$ having a protocol in place where patients are able to contact their provider if needed between telepsychiatry sessions

- $\quad$ ensuring that the video conferencing equipment is kept current in a manner where care is not disrupted and allows for thorough patient evaluation and follow up [22-23].

Certainly, challenges do exist as these standards will have difficulty being applied to international regions, remote areas of the globe without needed infrastructure, and paying attention to cultural norms when dealing with electronically based medical treatment. Furthermore, there must be increased emphasis on identifying cultural mores of patients and regions where these services are provided as they may not be readily apparent for a clinician in a geographically distant region. Moreover, there needs to be investment in logistical software which would allow clinicians to provide test instruments and other diagnostic assessment tools.

Additionally, reimbursement of telepsychiatry services continue to be in vague and stronger standards regarding these protocols would make if financially lucrative for clinicians, which in turn would lead to more access for patients requiring this service. In the end, as long as this medium remains a viable mechanism for patients to access care in a satisfactory manner, there will be a greater need for telepsychiatry.

\section{Conclusion}

The field of telepsychiatry has grown exponentially from its humble beginnings in the 1960s and continues to lead the way in terms of how medical care can be delivered. Psychiatry is a natural conduit due to its reliance on a much more verbal medium of evaluation and less invasive physical screening and treatment modalities compared to other medical disciplines. As the costs associated with medical care, especially in the United States, keep increasing, telepsychiatry is a field where the combination of effective diagnosis and treatment coupled with its cost-effective economics can lead to positive health outcomes. Furthermore, increased access to mental health providers in a timely fashion has the potential to benefit society as a whole with reduced costs of housing people who have had the mental condition worsened due to lack of seeing a provider. In addition, there continues to be potential in exporting this modality of treatment all over the world and even beyond; the possibility even exists of astronauts in the International Space Station receiving thorough mental health services if indicated. Finally, the combination of cost effectiveness combined with good care has the potential to further revolutionize the medical care landscape in the foreseeable future.

\section{Acknowledgment}

None.

\section{Conflict of interest}

No conflict of interest

\section{References}

1. Saeed SA, Johnson TL, Bagga M, Glass O (2016) Training Residents in the Use of Telepsychiatry: Review of the Literature and a Proposed Elective. Psychiatr Q 88(2): 271-283.

2. Bishop TF, Seirup JK, Pincus HA, Ross JS (2016) Population of US Practicing Psychiatrists Declined, 2003-13, Which May Help Explain Poor Access to Mental Health Care. Health Aff (Millwood) 35(7): 12711277

3. Pietrzak R, Johnson D, Goldstein M, Malley J, Southwick S (2009) Perceived Stigma and Barriers to Mental Health Care Utilization among OEF-OIF Veterans. Psychiatr Serv 60(8): 11182-11122.

4. Myers K \& Pruitt D (2016) A Road Map to Establish and Sustain a Telepsychiatry Practice. Journal of the American Academy of Child \& Adolescent Psychiatry 55(10): S42.

5. Grady B (2012) Promises and limitations of telepsychiatry in rural adult mental health care. World Psychiatry 11(3): 199-201.

6. Backhaus A, Agha Z, Maglione ML, Repp A, Ross B, et al. (2012) Videoconferencing psychotherapy: A systematic review. Psychol Serv 9(2): 111-131.

7. Morgan RD, Patrick AR, Magaletta PR (2008) Does the use of telemental health alter the treatment experience? Inmates' perceptions of telemental health versus face-to-face treatment modalities. J Consult Clin Psycho 76(1): 158-162.

8. Deslich S (2013) Telepsychiatry in Correctional Facilities: Using Technology to Improve Access and Decrease Costs of Mental Health Care in Underserved Populations. Perm J 17(3): 80-86.

9. Myers K, Cain S (2008) Practice Parameter for Telepsychiatry with Children and Adolescents. J Am Acad Child Adolesc Psychiatry 47(12): 1468-1483. 
10. Shore JH (2013) Telepsychiatry: Videoconferencing in the Delivery of Psychiatric Care. Am J Psychiatry 170(3): 256-262.

11. New York State Office of Mental Health (2015) Telepsychiatry standards guidance, USA.

12. American Telemedicine Association (2013) Practice guidelines for video-based online mental health services, USA.

13. American Telemedicine Association (2015) Who is ATA? USA.

14. Ngui EM, Khasakhala L, Ndetei D, Roberts LW (2010) Mental disorders, health inequalities and ethics: A global perspective. Int Rev Psychiatry 22(3): 235-244.

15. Chung Do J, Helm S, Fukuda M, Alicata D, Nishimura S, et al. (2012) Rural Mental Health: Implications for Telepsychiatry in Clinical Service, Workforce Development, and Organizational Capacity. Telemed J E Health 18(3): 244-246.

16. Leigh H, Cruz H, Mallios R (2009) Telepsychiatry appointments in a continuing care setting: Kept, cancelled and no-shows. J Telemed Telecare 15(6): 286-289.
17. Chan S, Parish M, Yellowlees P (2015) Telepsychiatry Today. Curr Psychiatry Rep 17(11): 89.

18. Voinescu BI, Szentagotai A, Coogan A (2010) Attitudes towards Psychiatry: A Survey of Romanian Medical Residents. Acad Psychiatry 34(1): 75-78.

19. Ziller EC, Anderson NJ, Coburn AF (2010) Access to Rural Mental Health Services: Service Use and Out-of-Pocket Costs. J Rural Health 26(3): 214224.

20. American Psychiatric Association (2016) Telepsychiatry Toolkit, USA.

21. American Psychiatric Association (2016) E-Mental health use expanding, showing promise, USA.

22. (2016) Telepsychiatry: The New Frontier in Mental Health.

23. Chong J \& Moreno FA (2102) Feasibility and acceptability of clinic-based telepsychiatry for low-income Hispanic primary care patients. Telemed J E Health 18(4): 297-304. 